\title{
Monitored Anesthesia Care versus General Anesthesia for Transcatheter Aortic Valve Replacement
}

\author{
Henry Holmes ${ }^{1}$, Matheus Falasa ${ }^{2}$, Dan $\mathrm{Neal}^{2}$, Calvin $\mathrm{Choi}^{2}$, Ki Park ${ }^{2}$, Anthony Bavry ${ }^{3}$, \\ Kirsten Freeman ${ }^{1}$, Eddie Manning ${ }^{2}$, Wade Stinson ${ }^{2}$, and Eric Jeng ${ }^{2}$ \\ ${ }^{1}$ University of Florida \\ ${ }^{2}$ University of Florida Health \\ ${ }^{3} \mathrm{UT}$ Southwestern
}

July 1, 2021

\begin{abstract}
Background: Monitored Anesthesia Care (MAC) has been increasingly used in lieu of general anesthesia (GA) for transcatheter aortic valve replacement (TAVR). We sought to compare outcomes and in-hospital costs between MAC and GA for TAVR at a single center Veterans Affairs Medical Center. Methods: A single-center retrospective review of 349 patients who underwent TAVR (MAC, $\mathrm{n}=244$ vs. GA, $\mathrm{n}=105$ ) from January 2014 to December 2019 was performed. Baseline patient characteristics, operating room (OR) time, intensive care unit (ICU) length of stay (LOS) and cost, total LOS, hospital cost, total cost, and complication rates were collected. Propensity matching was performed and resulted in 83 matched pairs. Results: In the unmatched TAVR cohort, MAC TAVR was associated with reduced OR time (146 vs. 198 minutes, P < .0001), ICU LOS $(1.4$ vs. 1.8 days, $\mathrm{P}<0.0001)$, total hospital $\operatorname{LOS}(3.4$ vs. 5.4 days, $\mathrm{P}<.0001)$, and lower index total cost $(\$ 81,3000$ vs. $\$ 85,400$, $\mathrm{P}=.010)$. After propensity matching, MAC TAVR patients had reduced OR time (146 vs. 196 minutes, P $<0.05)$, ICU LOS (1.2 vs. 1.7 days, $\mathrm{P}=.006)$, total $\operatorname{LOS}(3.5$ vs. 5.1 days, $\mathrm{P}=.001)$, and 180 -day mortality $(2.4 \%$ vs. $12 \%, \mathrm{P}<0.03)$. There was no difference in total hospitalization cost or total cost. Conclusions: In propensity matched groups, TAVR utilizing MAC is associated with improved OR time efficiency, decreased LOS, and a reduction in 180-day mortality, but no significant difference in cost.
\end{abstract}

\section{TITLE PAGE}

Title: Monitored Anesthesia Care versus General Anesthesia for Transcatheter Aortic Valve Replacement

Running head: Comparing Anesthesia Modalities for TAVR

\section{Authors:}

Henry R. Holmes, BS ${ }^{1}$; Matheus Falasa, $\mathrm{MD}^{1}$; Dan Neal, $\mathrm{MS}^{2}$; Calvin Y. Choi, MD, MS ${ }^{3,5}$; Ki Park, MD ${ }^{3,5}$; Anthony A. Bavry, MD, $\mathrm{MPH}^{6}$; Kirstin Freeman, $\mathrm{MD}^{1,4}$, Eddie W. Manning, MD ${ }^{1,4}$; Wade W. Stinson, $\mathrm{MD}^{1,4}$;; Eric I. Jeng, $\mathrm{MD}^{1}$.

\section{Institutions and Affiliations:}

University of Florida Health, 1600 SW Archer Rd, Gainesville, FL 32610 USA

${ }^{1}$ Division of Thoracic and Cardiovascular Surgery, Department of Surgery

${ }^{2}$ Department of Surgery

${ }^{3}$ Division of Cardiology, Department of Medicine 
North Florida/South Georgia Veterans Health System, 1601 SW Archer Rd, Gainesville, FL 32608 USA

${ }^{4}$ Division of Thoracic and Cardiovascular Surgery, Department of Surgery

${ }^{5}$ Division of Cardiology, Department of Medicine

UT Southwestern Medical Center, 2001 Inwood Rd, Dallas, TX 75390 USA

${ }^{6}$ Department of Internal Medicine, Division of Cardiology

Meeting Presentation: Presented at the 57th Annual Meeting of The Society of Thoracic Surgeons, Virtual Meeting, January 29-31, 2021.

Word Count: 3110

\title{
Corresponding Author:
}

Eric I. Jeng, MD, MBA

Assistant Professor of Surgery

Division of Thoracic and Cardiovascular Surgery

1600 SW Archer Rd, Gainesville, FL 32607

310-922-4265 (Mobile)

352-273-5501 (Office)

352-273-5513 (Facsimile)

eric.jeng@surgery.ufl.edu

\begin{abstract}
Background: Monitored Anesthesia Care (MAC) has been increasingly used in lieu of general anesthesia (GA) for transcatheter aortic valve replacement (TAVR). We sought to compare outcomes and in-hospital costs between MAC and GA for TAVR at a single center Veterans Affairs Medical Center. Methods: A single-center retrospective review of 349 patients who underwent TAVR (MAC, n $=244$ vs. GA, n $=105$ ) from January 2014 to December 2019 was performed. Baseline patient characteristics, operating room (OR) time, intensive care unit (ICU) length of stay (LOS) and cost, total LOS, hospital cost, total cost, and complication rates were collected. Propensity matching was performed and resulted in 83 matched pairs.

Results: In the unmatched TAVR cohort, MAC TAVR was associated with reduced OR time (146 vs. 198 minutes, $P<.0001)$, ICU LOS (1.4 vs. 1.8 days, $P<0.0001)$, total hospital LOS (3.4 vs. 5.4 days, $P<$ $.0001)$, and lower index total cost ( $\$ 81,3000$ vs. $\$ 85,400, P=.010)$. After propensity matching, MAC TAVR patients had reduced OR time (146 vs. 196 minutes, $P<0.05$ ), ICU LOS $(1.2$ vs. 1.7 days, $P=.006)$, total LOS (3.5 vs. 5.1 days, $P=.001$ ), and 180 -day mortality ( $2.4 \%$ vs. $12 \%, P<0.03)$. There was no difference in total hospitalization cost or total cost.

Conclusions: In propensity matched groups, TAVR utilizing MAC is associated with improved OR time efficiency, decreased LOS, and a reduction in 180-day mortality, but no significant difference in cost.
\end{abstract}

Abstract Word Count: 256

\section{Introduction}

Transcatheter aortic valve replacement (TAVR) initially emerged as an alternative to surgical aortic valve replacement (SAVR) for patients not previously considered surgical candidates due to comorbidities. ${ }^{1}$ The technology was then applied to patients considered high-risk for complications after SAVR, with studies demonstrating non-inferiority. ${ }^{2}$ Subsequently, TAVR was studied in the intermediate-risk patients, and more recently low risk patients. ${ }^{3-5}$ TAVR volumes have increased every year from 2011 to 2019, and now exceed 
surgical aortic valve replacement (SAVR). ${ }^{5,} 6$ The high cost of TAVR devices and increased costs compared to SAVR have prompted concerns of cost efficacy. ${ }^{7-13}$ The use of monitored anesthesia care (MAC) has evolved as one potential strategy for cost savings. MAC has increasingly been employed as a safe and effective alternative to general anesthesia (GA), with the proportion of TAVR performed under MAC nearly doubling from $33 \%$ in 2016 to $64 \%$ in $2019 .{ }^{14,15}$

However, there have been concerns of adverse events during the use of MAC in TAVR. ${ }^{16}$ Early series noted conversion rates from MAC to GA as high as $17 \%$, at a time when surgical access of the femoral artery was frequently required. ${ }^{17-19}$ More recent studies examining the conversion rate from MAC to GA found fewer than one percent of cases planned for MAC required conversion to GA, with reasons for conversion including patient discomfort and procedural complications, such as post-deployment aortic dissection with rupture and pericardial effusion causing tamponade. ${ }^{20}$ On the other hand, the advantages of MAC are well-documented. Studies using propensity-matching to compare outcomes between anesthesia modalities in TAVR found MAC was associated with reductions in fluoroscopy, procedure, and LOS times, as well as an increase in the proportion of patients discharged home. ${ }^{21,}{ }^{22} \mathrm{~A}$ recent instrumental variable analysis of the Transcatheter Valve Therapy Registry revealed decreased in-hospital and 30-day mortality, shorter hospital length of stay (LOS), and higher proportion of discharges to home when TAVR was performed with MAC compared to GA. ${ }^{14}$ Prospective randomized trials have also demonstrated the safety of MAC for TAVR. ${ }^{23}$

Despite the promising clinical outcomes for TAVR with MAC, the cost effectiveness of the approach in lowerrisk patients has not been well documented. Several early single-center studies compared costs between modalities in TAVR and demonstrated cost-savings with MAC. ${ }^{14,24-26}$ None of these included patients deemed low surgical risk, for which TAVR gained FDA-approval in August 2019. ${ }^{27}$ Comorbidities have been associated with adverse outcomes in TAVR. ${ }^{28}$ Given temporal differences in surgical risk and anesthesia modality as trends in TAVR use change, it is difficult to separate the effect of anesthesia modality from the effect of comorbidities.

Patient outcomes after TAVR at Veteran Affair (VA) TAVR centers have been shown to compare favorably with benchmark data outside the VA. ${ }^{29}$ The relative uniformity of the VA patient population and prospectively-collected databases at our institution offered a unique study opportunity. We sought to compare clinical outcomes and cost data between both anesthesia modalities for patients undergoing transfemoral TAVR. This included more recent cases, in which TAVR was used in lower-risk patients with fewer comorbidities.

\section{Patients and Methods}

Institutional review board approval was obtained prior to undertaking this study. All percutaneous transfemoral TAVR cases performed at the North Florida/South Georgia Veterans Affairs Medical Center (NF/SG VAMC) from January 2014 to December 2019 were retrospectively reviewed. TAVR patients were identified through a query of the local Veterans Affairs Surgery Quality Improvement Program (VASQIP) database, which contains baseline patient characteristics and clinical outcomes data for every cardiac surgical procedure performed. Valve-in-valve procedures and cases with missing cost data were excluded. The valve types changed with the evolving technology. The Edwards Sapien and Sapien XT valves used primarily between 2014-2015, and Edwards Sapien 3 from 2016-2019. The Medtronic Evolut R and Evolut Pro were used in a minority of cases. Patients were categorized by anesthesia type into MAC versus GA groups based on the utilization of endotracheal intubation. The decision to administer MAC or GA for each case was made in a multidisciplinary team format which included input from anesthesiologists, cardiologists, and cardiac surgeons. The analysis was performed on an as-treated basis.

Demographics, baseline medical history, and outcome data points were collected according to VASQIP definitions. Diabetes was defined as any outpatient preoperative hyperglycemia requiring oral medications or insulin. History of cardiac surgery was defined as any history of cardiac surgery, on or off pump, such as coronary artery bypass graft, valve replacement, or other procedures. Preoperative atrial fibrillation was defined as the presence of atrial fibrillation or atrial flutter in the two weeks preceding surgery. Preoperative 
myocardial infarction (MI) was defined as any prior history of MI, regardless of timeframe, prior to surgery. Thirty-day postoperative morbidity and mortality estimates were calculated by the VASQIP risk score.

Complications and outcomes were defined as follows: postoperative renal failure was defined as the development of new renal failure requiring renal replacement therapy, or an exacerbation of preoperative renal failure requiring initiation of renal replacement therapy within 30 days postoperatively. Postoperative atrial fibrillation included new-onset atrial fibrillation or atrial flutter requiring any treatment. Postoperative MI included any MI that occurred within 30 days postoperatively. Postoperative cerebrovascular accident (CVA) was defined as any new objective neurologic deficit lasting more than 72 hours with onset immediately post-operatively or occurring within the 30 days after surgery. Intraoperative cardiac arrest was defined as any cardiac arrest requiring external or open cardiopulmonary resuscitation (CPR) in the operating room, whereas postoperative cardiac arrest included any cardiac arrest requiring CPR within 30 days postoperatively. All lab values represent the results reported closest to the date of the surgery and exclude any lab values collected beyond 30 days before surgery.

Direct cost data was derived from the local VA Managerial Cost Accounting system, which collects and stores direct cost data associated with every VA hospitalization. Costs were reported under the following categories: direct operating room (OR) cost, direct intensive care unit (ICU) cost, and overall direct costs. Direct costs are defined as costs directly associated with patient care, such as anesthesia services, nursing services, drugs, medical supplies, and hospitalization. Indirect costs, commonly referred to as overhead costs, encompass the expenses not directly tied to a patient's care, such as administrative expenses, medical records, and information services. Indirect costs were not analyzed in this study, and all costs subsequently mentioned are direct costs only. The types and costs of the valve prostheses used for each patient were extracted from a local VA prosthetics database. Total hospital cost was calculated, which included physician charges, labs, imaging, operating room, intensive care unit, step-down unit, and hospital floor costs. Overall cost included all these inpatient charges, plus the cost of the prosthetic valve.

A non-matched analysis was initially performed. Patient characteristics, ICU LOS, total hospital LOS, and costs were compared using Fisher's exact test for binary data and Wilcoxon rank sum test for continuous data. Propensity score matching was then performed. The R package "matchit" was used to match patients who received GA with those who received MAC according to their propensity for receiving either. To assess propensity, a logistic regression model with MAC as the outcome and VASQIP estimated mortality, age, sex, and BMI as covariates was used. History of diabetes, history of COPD, creatinine, and hemoglobin were excluded from the model because these factors are integrated into the VASQIP estimated mortality. Additionally, only preoperative variables were utilized, and no procedure-related variables, including postoperative echocardiogram findings (gradient, presence/absence of paravalvular leaks, postoperative ejection fraction), EKG changes and/or pacer requirement, were integrated. Patients were matched using a 1-to-1 matching with a caliper width of 0.1 (meaning that matched pairs were within 0.1 standard deviations of each other on propensity score). This process resulted in the matching of 83 of 105 GA recipients with 83 of 244 MAC recipients. To assess the effect of anesthesia type on outcomes, mixed-effects models (logistic or linear regression as appropriate) with anesthesia type as a fixed factor and a random factor for a matched pair were used to account for the dependency introduced by matching. For outcomes that were too sparse for a logistic regression model, McNemar's test for paired data was used. Finally, to assess whether OR times were significantly shorter with MAC due to the anesthesia modality itself or due to increased institutional experience (as MAC was used increasingly in later years), OR time was compared between MAC and GA groups for the year 2016 only. All analyses were performed using R (V.4.0.2, The R Foundation for Statistical Computing, Vienna, Austria).

\section{Results}

From January 2014 to December 2019, 356 elective percutaneous transfemoral TAVR procedures were performed at the NF/SG VAMC. Seven of these met exclusion criteria, leaving 349 patients included in the study. Of these, 244 cases were classified as MAC, and 105 as GA. Starting in 2015, there was a steep decline in the use of GA, and a rapid increase in the use of MAC (Figure 2). Baseline characteristics of 
the entire cohort are summarized in Table 1 . With unmatched data, the MAC group had higher mean BMI (30.4 vs. $\left.28.7 \mathrm{~kg} / \mathrm{m}^{2}, P=.010\right)$, higher average preoperative hemoglobin $(12.7$ vs. $12.2 \mathrm{~g} / \mathrm{dL}, P=$ $.031)$, and were less likely to have a history of prior cardiac surgery $(27.9 \%$ vs. $41.0 \%, P=.018)$. Patients who underwent TAVR with MAC had lower VASQIP estimated mortality $(2.18 \%$ vs. $6.11 \%, P<.001)$. All other patient characteristics were similar between the two groups. Outcomes between unmatched groups are summarized in Table 2. Despite patients in the MAC group having lower estimated VASQIP estimated mortality preoperatively, there was no difference in postoperative renal failure $(0 \%)$, postoperative MI $(0.4 \%$ vs. $1.9 \%, P=.216)$, postoperative cerebrovascular accident ( $0 \%$ vs. $1 \%, P=.301)$, postoperative atrial fibrillation ( $1.6 \%$ vs $3.8 \%, P=.248)$, postoperative cardiac arrest $(0.4 \%$ vs. $0 \%, P=1)$, and percentage of patients discharged to home $(97.1 \%$ vs. $96.3 \%, P=.628)$. Intraoperative cardiac arrest $(2.9 \%$ vs. $0.4 \%, P$ $=.083)$ and 30-day mortality ( $2.9 \%$ vs. $0.4 \%, P=.083)$, were not different between groups. The GA TAVR cohort had a significantly higher 180 -day mortality $(12.4 \%$ vs. $3.3 \%, P=.002)$. TAVR cases performed with MAC had shorter OR times (146 vs. 198 minutes, $P<.001$ ), shorter lengths of stay in the ICU (1.4 vs. 1.8 days, $P<.001)$ and shorter total hospital length of stay $(3.4$ vs. 5.4 days, $P<.001)$. The use of MAC was also associated with lower hospital cost $(\$ 48,800$ vs. $\$ 52,600, P<.001)$ and lower overall cost after valve cost was included $(\$ 81,300$ vs. $\$ 85,400, P<.001)$. Average ICU cost was $\$ 8,513$ for MAC and $\$ 11,161$ for GA $(P=.019)$. Mean OR costs were $\$ 35,994$ for MAC and $\$ 32,943$ for GA $(P=.024)$. The average costs of valves were $\$ 32,514$ for $\mathrm{MAC}$ and $\$ 32,843$ for $\mathrm{GA}(P=.178)$.

Propensity matching resulted in 83 MAC patients matched to 83 GA patients (Figure 1 ). Demographics and patient characteristics of the 83 matched pairs were compared (Table $\mathbf{3}$ ). Between matched pairs, there were no significant differences between patients who underwent MAC to those that received GA in age $(77.6$ years vs. 77 years, $P=.831)$, sex $(97.6 \%$ male, $P=1)$, BMI $\left(29.4\right.$ vs. $29.3 \mathrm{~kg} / \mathrm{m}^{2}, P=$ $.581)$, preoperative creatinine (1.4 vs. $1.2 \mathrm{mg} / \mathrm{dL}, P=.791)$, preoperative hemoglobin $(12.3 \mathrm{~g} / \mathrm{dL}, P=$ $.791)$, or VASQIP estimated mortality $(2.7 \%$ vs. $3.1 \%, P=.200)$. There were no differences between propensity-matched groups in the incidence of postoperative MI ( $0 \%$ vs. $2 \%, P=.480)$, renal failure requiring renal replacement therapy $(0 \%)$, intraoperative cardiac arrest $(1.2 \%$ vs. $1.2 \%, P=1)$, postoperative atrial fibrillation ( $1.2 \%$ vs. $3.6 \%, P=.335)$, postoperative CVA ( $1 \%$ vs. $1.2 \%, P=1)$, or frequency of discharge home $(96.4 \%$ vs. $97.4 \%, P=.780)$. The MAC TAVR cohort did have significant reductions in OR times (147 vs. 196 minutes, $P<.001)$, ICU LOS $(1.2$ vs. 1.7 days, $P=.006)$, total LOS (3.5 vs. 5.1 days, $P$ $=.001)$, and 180-day mortality (2.4\% vs. $12 \%, P=.030)$ when compared to the propensity-matched GA TAVR group (Table 4 ). There were no significant differences between matched groups in hospital costs $(\$ 49,700$ vs. $\$ 50,400, P=.709)$ or overall cost $(\$ 82,100$ vs. $\$ 83,100, P=.936)$.

OR time for all 2016 TAVR cases were compared by anesthesia modality to eliminate bias secondary to physician and institutional experience. There was a significant reduction in OR time when MAC was used for TAVR (150 vs. 201 minutes, $P<.001)$.

\section{Comment}

Unmatched analysis in this study demonstrates an association between the use of MAC for TAVR and reductions in OR time, ICU LOS, total LOS, hospital cost, and total cost. However, MAC was also associated with lower VASQIP estimated mortality (Table 1 ). This difference reflects temporal trends in TAVR use. TAVR was initially applied only to patients not considered surgical candidates as a salvage alternative to medical therapy, then to high risk patients, and eventually to intermediate and low risk patients in later years. ${ }^{2}$ 27, 30-32 MAC was not used for TAVR at this institution until 2015, and as it became increasingly implemented, the use of GA declined (Figure 2 ). This trend is consistent with national trends. ${ }^{14}$ Given the asymmetrical temporal distribution of surgical risk and anesthesia modalities with time, propensity matching was necessary to reduce confounding bias and for meaningful comparisons between anesthesia modalities for TAVR.

The incidence of adverse outcomes, including atrial fibrillation, cardiac arrest, CVA, MI, and renal failure requiring renal replacement therapy, were not significantly different between propensity-matched groups of TAVR patients. The incidence of these events after TAVR is known to be low, and this study was insufficiently 
powered to detect differences in the occurrence of these uncommon events. ${ }^{14} 25,29$, 33 No significant differences were found in 30-day mortality or the percentage of patients discharged elsewhere besides home, but MAC was associated with lower 180-day mortality. It is difficult to ascertain whether this is a true effect of the anesthesia modality or a reflection of underlying bias not eliminated by propensity matching. Studies have suggested 30-day mortality may underestimate outcomes, and longer-term outcomes may be better metrics for assessing hospital performance and patient outcomes. ${ }^{34}$

Several studies have compared costs between MAC and GA in TAVR. ${ }^{14,24,25,35-37}$ This study demonstrated significant differences between anesthesia modalities in OR time, ICU LOS, and total LOS after propensitymatching. It was hypothesized that these differences would translate to reductions in patient charges, but this was not the case. The absence of significant cost savings among MAC TAVR has been reported in a prior unmatched single-center study, which similarly found decreased ICU and hospital LOS with MAC but no cost benefit. ${ }^{36}$ The authors found similarity in costs was mostly driven by increased "miscellaneous" costs in the MAC group, which primarily included the costs of treating unrelated comorbidities during the hospital stay. A 2014 retrospective unmatched comparison between TAVR via "minimalist approach," involving MAC and the use of catheterization lab, and a "standard approach," which involved GA and the use of a hybrid OR, found the minimalist approach was associated with an average cost reduction of $\$ 9,892 .{ }^{24}$ The high cost of a hybrid OR likely explains these findings, and the use of MAC may not have driven savings. ${ }^{38,39}$ A 2017 propensity-matched analysis found a $28 \%$ decrease in direct cost when MAC was used for TAVR. ${ }^{25}$ However, this latter study was of high-risk patients, with a large percentage of cases being done by transapical approach. Average hospital stay for both arms was nearly double that observed in this series, highlighting the limited generalizability of the study to more recent trends in the use of TAVR. Other comparisons of high-risk patients, confounded by baseline differences between groups, have demonstrated reduced OR, ICU, and total hospital LOS times with the use of MAC for TAVR. ${ }^{35}$, ${ }^{37}$ With the inclusion of intermediate and low risk patients, the data presented herein offers more generalizability to the population currently treated with TAVR.

Hospital and operator volume have been demonstrated to correlate with outcomes in TAVR. ${ }^{40-42}$ To minimize the impact of physician and institutional experience on OR time, we compared MAC and GA TAVR within the year 2016. This year was chosen as it had the most even distribution of TAVR between MAC and GA and followed over 60 cases and two years of institutional experience with TAVR. In this analysis, MAC was still associated with significantly shorter OR time, suggesting the difference is due to the anesthesia modality itself, not confounders such as increased institutional experience or new deployment devices.

Physician experience and case volume vary considerably between studies, which poses a challenge when comparing our findings to previous analyses. Among the TAVR programs developed at 13 national VAMC's, our center is the highest volume VAMC TAVR program based on internal data. While prior studies were performed at non-VA hospitals, this is the first study comparing costs between MAC and GA TAVR at a VAMC, which has a very standardized cost structure. Differences in administrative, clinical, and billing operations between non-VA and VA centers may limit extrapolation of costs to non-VA centers. For instance, the relative value units (RVU) used for reimbursement purposes are calculated differently between VAMCs and other hospitals. RVUs defined by the Centers for Medicare and Medicaid Services (CMS) for non-VA facilities are based on factors like technical skill, stress, and judgement required for a treatment associated with a current procedural terminology (CPT) code. The VA instead uses a unique RVU formula that is based on the resources used for a service (e.g., OR time, ICU LOS) and further specifies RVUs for several different resource categories (labor, supplies, overhead, contractors, etc.) ${ }^{43}$ It can be difficult to extrapolate costs between VA and non-VA hospitals, as studies of these differences are limited. Healthcare costs for end-stage renal disease patients have been shown to be more expensive at VA hospitals compared to at non-VA hospitals, but studies like this are confounded by comorbidity distribution and different approaches to medical management. ${ }^{44}$ More recent comparisons have demonstrated increased costs at VA hospitals compared to community hospitals for coronary artery bypass grafting, but lower costs for percutaneous coronary intervention. ${ }^{45}$ Cost data for other hospitals would include indirect costs, such as administrative expenses. 
While our study was performed at the highest volume VA TAVR program, it remains limited by the propensity matched sample size and its retrospective nature. Additionally, the VA patient population is almost exclusively male, limiting the generalizability of our results. Despite these limitations, our study demonstrates strong advantages to the use of MAC for TAVR. In a high volume VAMC, TAVR performed under MAC is safe, leads to an improvement in OR efficiency, and reduces ICU and hospital length of stay, but does not translate into cost savings.

\section{ACKNOWLEDGEMENTS AND DISCLOSURES}

This work was not supported by any grants or other financial support. The authors disclose no conflicts of interests.

\section{REFERENCES}

1. Leon MB, Smith CR, Mack M, et al. Transcatheter aortic-valve implantation for aortic stenosis in patients who cannot undergo surgery. $N$ Engl J Med 2010; 363(17):1597-607.

2. Smith CR, Leon MB, Mack MJ, et al. Transcatheter versus surgical aortic-valve replacement in high-risk patients. N Engl J Med2011; 364(23):2187-98.

3. Popma JJ, Deeb GM, Yakubov SJ, et al. Transcatheter Aortic-Valve Replacement with a Self-Expanding Valve in Low-Risk Patients. N Engl J Med 2019; 380(18):1706-1715.

4. Mack MJ, Leon MB, Thourani VH, et al. Transcatheter Aortic-Valve Replacement with a BalloonExpandable Valve in Low-Risk Patients. N Engl J Med 2019; 380(18):1695-1705.

5. Carroll JD, Mack MJ, Vemulapalli S, et al. STS-ACC TVT Registry of Transcatheter Aortic Valve Replacement. Ann Thorac Surg 2021; 111(2):701-722.

6. Boskovski MT, Nguyen TC, Mccabe JM, et al. Outcomes of Transcatheter Aortic Valve Replacement in Patients With Severe Aortic Stenosis.JAMA Surgery 2020; 155(1):69.

7. Azraai M, Gao L, Ajani AE. Cost-Effectiveness of Transcatheter Aortic Valve Intervention (TAVI) Compared to Surgical Aortic Valve Replacement (SAVR) in Low- to Intermediate-Surgical-Risk Patients. Cardiovasc Revasc Med 2020; 21(9):1164-1168.

8. Baron SJ, Wang K, House JA, et al. Cost-Effectiveness of Transcatheter Versus Surgical Aortic Valve Replacement in Patients With Severe Aortic Stenosis at Intermediate Risk. Circulation 2019; 139(7):877-888.

9. Fairbairn TA, Meads DM, Hulme C, et al. The cost-effectiveness of transcatheter aortic valve implantation versus surgical aortic valve replacement in patients with severe aortic stenosis at high operative risk. Heart 2013; 99(13):914-20.

10. Reynolds MR, Lei Y, Wang K, et al. Cost-Effectiveness of Transcatheter Aortic Valve Replacement With a Self-Expanding Prosthesis Versus Surgical Aortic Valve Replacement. J Am Coll Cardiol 2016; 67(1):29-38.

11. Zhou JY, Liew D, Duffy SJ, et al. Cost-Effectiveness of Transcatheter Versus Surgical Aortic Valve Replacement in Low-Risk Patients With Severe Aortic Stenosis. Heart Lung Circ 2021; 30(4):547-554.

12. Tam DY, Hughes A, Fremes SE, et al. A cost-utility analysis of transcatheter versus surgical aortic valve replacement for the treatment of aortic stenosis in the population with intermediate surgical risk. $J$ Thorac Cardiovasc Surg 2018; 155(5):1978-1988.e1.

13. Manolis AS. Transcatheter aortic valve implantation economics: a grisly reality. Annals of Cardiothoracic Surgery 2017; 6(5):516-523.

14. Butala NM, Chung M, Secemsky EA, et al. Conscious Sedation Versus General Anesthesia for Transcatheter Aortic Valve Replacement: Variation in Practice and Outcomes. JACC Cardiovasc Interv 2020; 13(11):1277-1287. 
15. Fröhlich GM, Lansky AJ, Webb J, et al. Local versus general anesthesia for transcatheter aortic valve implantation (TAVR)-systematic review and meta-analysis. BMC medicine 2014; 12:41-41.

16. Mayr NP, Wiesner G, Husser O, et al. Critical adverse events during transfemoral TAVR in conscious sedation. Is an anesthesiologic support mandatory? Cardiovascular Revascularization Medicine 2018; 19(6):41-46.

17. Ben-Dor I, Looser PM, Maluenda G, et al. Transcatheter aortic valve replacement under monitored anesthesia care versus general anesthesia with intubation. Cardiovasc Revasc Med 2012; 13(4):207-10.

18. Bergmann L, Kahlert P, Eggebrecht H, et al. Transfemoral aortic valve implantation under sedation and monitored anaesthetic care-a feasibility study. Anaesthesia 2011; 66(11):977-82.

19. Brecker SJ, Bleiziffer S, Bosmans J, et al. Impact of Anesthesia Type on Outcomes of Transcatheter Aortic Valve Implantation (from the Multicenter ADVANCE Study). Am J Cardiol 2016; 117(8):1332-8.

20. Kimmel J, Potosky R, Williams MR, et al. Conversion from Monitored Anesthesia Care to General Anesthesia for Transcatheter Aortic Valve Replacement. J Cardiothorac Vasc Anesth 2018; 32(2):1032-1040.

21. Kislitsina ON, Smith D, Sherwani SS, et al. Comparison of Monitored Anesthesia Care and General Anesthesia for Transcatheter Aortic Valve Replacement. Innovations (Phila) 2019; 14(5):436-444.

22. Abbett SK, Urman RD, Resor C, et al. The Impact of Anesthesia Type on Outcomes in Patients Undergoing Transcatheter Aortic Valve Replacement. Journal of Cardiothoracic and Vascular Anesthesia2020.

23. Thiele H, Kurz T, Feistritzer H-J, et al. General versus Local Anesthesia with Conscious Sedation in Transcatheter Aortic Valve Implantation: The Randomized SOLVE-TAVI Trial. Circulation 2020.

24. Babaliaros V, Devireddy C, Lerakis S, et al. Comparison of Transfemoral Transcatheter Aortic Valve Replacement Performed in the Catheterization Laboratory (Minimalist Approach) Versus Hybrid Operating Room (Standard Approach). JACC: Cardiovascular Interventions2014; 7(8):898-904.

25. Toppen W, Johansen D, Sareh S, et al. Improved costs and outcomes with conscious sedation vs general anesthesia in TAVR patients: Time to wake up? PloS one 2017; 12(4):e0173777-e0173777.

26. Mosleh W, Mather JF, Amer MR, et al. Propensity Matched Analysis Comparing Conscious Sedation Versus General Anesthesia in Transcatheter Aortic Valve Implantation. Am J Cardiol 2019; 124(1):70-77.

27. Coylewright M, Forrest JK, McCabe JM, et al. TAVR in Low-Risk Patients: FDA Approval, the New NCD, and Shared Decision-Making. J Am Coll Cardiol 2020; 75(10):1208-1211.

28. Feldman D, Romashko M, Koethe B, et al. COMORBIDITY BURDEN AND ADVERSE OUTCOMES AFTER TRANSCATHETER AORTIC VALVE REPLACEMENT. Journal of the American College of Cardiology 2020; 75(11):1423.

29. Hall PS, O'Donnell CI, Mathew V, et al. Outcomes of Veterans Undergoing TAVR Within Veterans Affairs Medical Centers: Insights From the Veterans Affairs Clinical Assessment, Reporting, and Tracking Program. JACC Cardiovasc Interv 2019; 12(21):2186-2194.

30. Mack MJ, Leon MB, Smith CR, et al. 5-year outcomes of transcatheter aortic valve replacement or surgical aortic valve replacement for high surgical risk patients with aortic stenosis (PARTNER 1): a randomised controlled trial. Lancet 2015; 385(9986):2477-84.

31. Anantha-Narayanan M, Kandasamy VV, Reddy YN, et al. Low-Risk Transcatheter Versus Surgical Aortic Valve Replacement - An Updated Meta-Analysis of Randomized Controlled Trials. Cardiovasc Revasc Med 2020; 21(4):441-452.

32. Ando T, Ashraf S, Villablanca P, et al. Meta-Analysis of Effectiveness and Safety of Transcatheter Aortic Valve Implantation Versus Surgical Aortic Valve Replacement in Low-to-Intermediate Surgical Risk Cohort. Am J Cardiol 2019; 124(4):580-585. 
33. Okoh AK, Haik N, Haik B, et al. Periprocedural complications after transcatheter aortic valve replacement and their impact on resource utilization. Cardiovascular Revascularization Medicine 2020.

34. Hirji S, Mcgurk S, Kiehm S, et al. Utility of 90-Day Mortality vs 30-Day Mortality as a Quality Metric for Transcatheter and Surgical Aortic Valve Replacement Outcomes. JAMA Cardiology 2020; 5(2):156.

35. Sammour Y, Kerrigan J, Banerjee K, et al. Comparing outcomes of general anesthesia and monitored anesthesia care during transcatheter aortic valve replacement: The Cleveland Clinic Foundation experience. Catheter Cardiovasc Interv 2021.

36. Ahmad M, Patel JN, Vipparthy SC, et al. Conscious Sedation Versus General Anesthesia in Transcatheter Aortic Valve Replacement: A Cost and Outcome Analysis. Cureus 2019.

37. Lau W, Shannon F, Hanzel GS, et al. OUTCOME-BASED COST ANALYSIS OF TRANSFEMORAL TRANSCATHETER AORTIC VALVE REPLACEMENT USING FASCIA ILIACA COMPARTMENT BLOCK AND MINIMALIST CONSCIOUS SEDATION APPROACH VERSUS GENERAL ANESTHESIA. Journal of the American College of Cardiology2017; 69(11):1330.

38. Patel S, Lindenberg M, Rovers MM, et al. Understanding the Costs of Surgery: A Bottom-Up Cost Analysis of Both a Hybrid Operating Room and Conventional Operating Room. Int J Health Policy Manag 2020 .

39. Potter BJ, Ann Thompson C. Cost of operating room time for endovascular transcatheter aortic valve replacement. J Med Econ2019; 22(10):1022-1024.

40. Rong LQ, Gaudino M, Tam D, et al. Impact of operator characteristics on outcomes in transcatheter aortic valve replacement. The Annals of Thoracic Surgery 2020.

41. Carroll JD, Vemulapalli S, Dai D, et al. Procedural Experience for Transcatheter Aortic Valve Replacement and Relation to Outcomes. Journal of the American College of Cardiology 2017; 70(1):29-41.

42. Khera S, Kolte D, Gupta T, et al. Association Between Hospital Volume and 30-Day Readmissions Following Transcatheter Aortic Valve Replacement. JAMA Cardiology 2017; 2(7):732.

43. Nugent GN, Hendricks A, Nugent L, et al. Value for taxpayers' dollars: what VA care would cost at medicare prices. Med Care Res Rev 2004; 61(4):495-508.

44. Hynes DM, Stroupe KT, Fischer MJ, et al. Comparing VA and private sector healthcare costs for endstage renal disease. Med Care2012; 50(2):161-70.

45. Barnett PG, Hong JS, Carey E, et al. Comparison of Accessibility, Cost, and Quality of Elective Coronary Revascularization Between Veterans Affairs and Community Care Hospitals. JAMA Cardiol 2018; 3(2):133141.

\section{Hosted file}

MACvGA.Tables and Figures.V2.docx available at https://authorea.com/users/423138/articles/ 528676-monitored-anesthesia-care-versus-general-anesthesia-for-transcatheter-aorticvalve-replacement 